\title{
Anterior peri-insular quadrantotomy: a cadaveric white matter dissection study
}

\author{
*Pablo Gonzalez-Lopez, MD, PhD, ${ }^{1}$ Giulia Cossu, MD, ${ }^{2}$ Etienne Pralong, MD, ${ }^{2}$ \\ Matias Baldoncini, MD, ${ }^{3}$ Mahmoud Messerer, MD, MSc, ${ }^{2,4}$ and Roy Thomas Daniel, MD, MCh $^{2,4}$ \\ 'Department of Neurosurgery, Hospital General Universitario Alicante, Spain; ${ }^{2}$ Department of Neurosurgery, University Hospital \\ of Lausanne, Switzerland; ' ${ }^{3}$ Department of Neurological Surgery, San Fernando Hospital, Buenos Aires, Argentina; and ${ }^{4}$ Faculty \\ of Medicine and Biology, University of Lausanne, Switzerland
}

\begin{abstract}
OBJECTIVE Anterior quadrant disconnection represents a safe surgical option in well-selected pediatric patients with a large frontal lobe lesion anterior to the motor cortex. The understanding of the anatomy of the white matter tracts connecting the frontal lobe with the rest of the cerebrum forms the basis of a safe and successful disconnective surgery. The authors explored and illustrated the relevant white matter tracts sectioned during each surgical step using fiber dissection techniques.
\end{abstract}

METHODS Five human cadaveric hemispheres were dissected to illustrate the frontal connections in the 3 planes. The dissections were performed from lateral to medial, medial to lateral, and ventral to dorsal to describe the various tracts sectioned during the 4 steps of this surgery, namely the anterior suprainsular window, intrafrontal disconnection, anterior callosotomy, and frontobasal disconnection.

RESULTS At the beginning of each surgical step, the $U$ fibers were cut. During the anterior suprainsular window, the superior longitudinal fasciculus (SLF), the uncinate fasciculus, and the inferior fronto-occipital fasciculus (IFOF) were visualized and sectioned, followed by sectioning of the anterior limb of the internal capsule. During the intrafrontal disconnection, the SLF was cut, along with the corona radiata. At the medial surface the cingulum was sectioned. The anterior callosotomy disconnected the anterior third of the body of the callosum, the genu, and the rostrum. The frontobasal disconnection addressed the last remaining fibers connecting the frontal lobe with the rest of the hemisphere, namely the anterior limb of the anterior commissure.

CONCLUSIONS The anterior peri-insular quadrantotomy aims at effectively treating children with large lesions of the frontal lobe anterior to the motor cortex. A precise understanding of the gyral anatomy of this lobe along with the several white matter connections is crucial to avoid motor complications and to ensure complete disconnection.

https://thejns.org/doi/abs/10.3171/2019.10.PEDS19472

KEYWORDS epilepsy surgery; anterior quadrantotomy; disconnective surgery; frontal lobe

$\mathrm{F}$ ocal epilepsies restricted to the frontal lobe account for $20 \%$ of patients with refractory epilepsy, and they represent the second most common cause for focal epilepsy syndromes after temporal lobe epilepsy. ${ }^{10,11}$ Small lesions are treated by simple lesionectomy, while larger lesions have been traditionally treated by frontal lobectomy. ${ }^{3,8}$ Disconnective surgery, such as hemispherotomy or anterior and/or posterior quadrantotomy, has gained increasing acceptance for treating the large hemispheric and/or subhemispheric lesions that are frequently seen in the pediatric population. ${ }^{3-6}$

The anterior quadrant is defined as the portion of the frontal lobe anterior to the precentral gyrus and is composed of the superior, middle, and inferior frontal gyri on the lateral surface of the cerebrum, the cingulate gyrus and the anterior part of the callosum on the medial surface, and the orbital gyri and gyrus rectus on the basal surface. Patients with well-defined epileptogenic lesions of the frontal lobe and with sparing of the perirolandic cortex and preserved motor functions are ideal candidates for an anterior peri-insular quadrantotomy that keeps the motor cortex and the posterior quadrant and their projections intact, thereby maintaining neurological functions. Disconnective surgery avoids the classical complications

ABBREVIATIONS IFOF = inferior fronto-occipital fasciculus; SLF = superior longitudinal fasciculus. SUBMITTED August 12, 2019. ACCEPTED October 21, 2019.

${ }^{*}$ P.G.L. and G.C. contributed equally to this work. 
associated with large brain resections. ${ }^{3,11}$ We recently published the technical details of this surgery along with a case description of a 9-year-old child. ${ }^{2}$ Due to the rarity of these cases, reports from the literature on this group of patients are scarce.

We performed a cadaveric study that details the anatomy of the white matter fibers that are disconnected during the isolation of the frontal lobe from the rest of the cerebrum during the different surgical steps of this procedure.

\section{Methods}

Five human brain specimens were prepared according to Klingler's technique for white matter dissection. ${ }^{10}$ Thus, the brains were extracted when they were as fresh as possible, during the first 12 hours postmortem. The specimens were cleaned under running water and fixed for 8 weeks in a $10 \%$ formaldehyde solution. Each piece underwent fixation while hanging upside down by a suture in the basilar artery to avoid morphological disruptions of its shape and size.

After the 8-week fixation, the specimens were cleaned under running water again and then frozen to $-15^{\circ} \mathrm{C}$ for 2 weeks. The brains were then thawed and the arachnoid tissue and vessels were carefully dissected and detached from the cortical surface. After this 10 -week period, the specimens were ready for the dissection. All the specimens were dissected from the lateral, medial, and basal aspects of each frontal lobe, with special attention paid to the sequential removal of underlying white matter tracts. The target in all 3 areas was reaching the frontal horn of the ipsilateral lateral ventricle.

Each step of the dissection was photographed to produce high-definition white matter dissection images to show the different tracts exposed, which are those disconnected during the anterior peri-insular quadrantotomy. Special attention was given to the different connections of all these tracts to determine whether this original surgical procedure allows a complete anterior quadrant disconnection.

\section{Results}

Anatomically, the anterior quadrant is limited posteriorly by the precentral sulcus on the lateral surface and by the paracentral ascending branch of the cingulate sulcus on the medial surface (Fig. 1A). Below the surface, the anterior quadrant is limited by the anterior part of the circular sulcus and by the anterior perforated substance.

As already detailed in our previous report, ${ }^{2}$ the peri-insular anterior quandrantotomy can be divided into 4 surgical steps: 1) the anterior suprainsular window, 2) the anterior callosotomy, 3) the intrafrontal disconnection, and 4) the frontobasal disconnection. The details of these disconnections are illustrated in Fig. 1. The following paragraphs detail the procedure as performed in the cadaver dissection to illustrate the white matter tracts encountered and sectioned during this disconnective procedure.

\section{Lateral Dissection}

Lateral to medial dissection was used to progressively
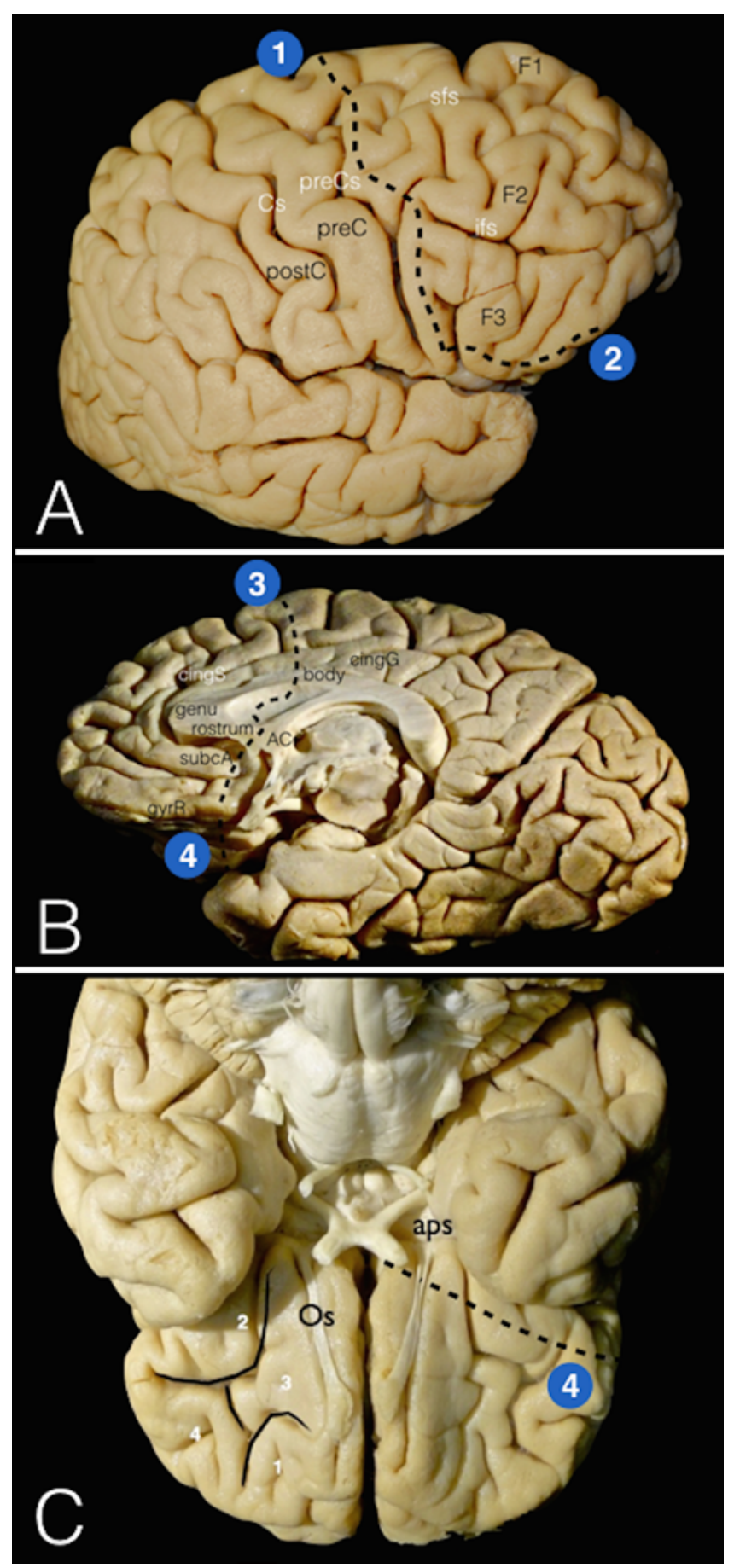

FIG. 1. High-definition white matter dissection images of a righthemisphere brain specimen in which the 4 sequential corticotomies are represented. A: Lateral view of the right hemisphere. The first corticotomy (1) runs parallel and anterior to the precentral sulcus, disrupting the cerebral cortex of the posterior aspects of F1, F2, and F3. The second corticotomy (2) represents the laterobasal disconnection parallel to the floor of the anterior fossa through the basal aspect of F3. B: Mediobasal view of the right cerebral hemisphere showing the medial (3) and basal disconnections (4). The third corticotomy (3) disrupts the cortex of the medial gyrus of the frontal lobe as well as the cingulum and corpus callosum. FIG. 1. (continued) $\rightarrow$ 
FIG. 1. C: Basal aspect of the cerebral hemisphere, where the basal corticotomy is represented, disrupting the cortical aspect of the lateral, posterior, and medial fronto-orbital gyri and gyrus rectus: 1, anterior fronto-orbital gyrus; 2, posterior fronto-orbital gyrus; 3 , medial fronto-orbital gyrus; 4 , lateral fronto-orbital gyrus. For the surgical procedure in patients, the skin incision performed is a barn door-type incision, placed in the frontotemporal region, that allows access to perform a frontotemporoparietal craniotomy. This allows adequate exposure of the anterior sylvian point, superior temporal gyrus, inferior and middle frontal gyri, and precentral gyrus (shown in panel A). The cortical incision is placed in the frontal operculum parallel to the sylvian fissure, and this incision is turned superiorly at its posterior end to perform the intrafrontal disconnection. These incisions are deepened to the ventricle, where the anterior callosotomy is performed. The intrafrontal disconnection is then continued medially to reach the interhemispheric fissure by disruption of the frontal cortices at its medial surface (shown in panel B). AC = anterior commissure; aps = anterior perforated substance; body = body of the corpus callosum; cing $=$ cingulate gyrus; cingS = cingulate sulcus; $C s=$ central sulcus; F1 = superior frontal gyrus; F2 = middle frontal gyrus; F3 = inferior frontal gyrus; genu = genu of the corpus callous; gyrR = gyrus rectus; ifs = inferior frontal sulcus; Os = olfactory sulcus; postC = postcentral gyrus; preC = precentral gyrus; preCs = precentral sulcus; rostrum = rostrum of the corpus callosum; sfs = superior frontal sulcus; subcA = subcallosal area.
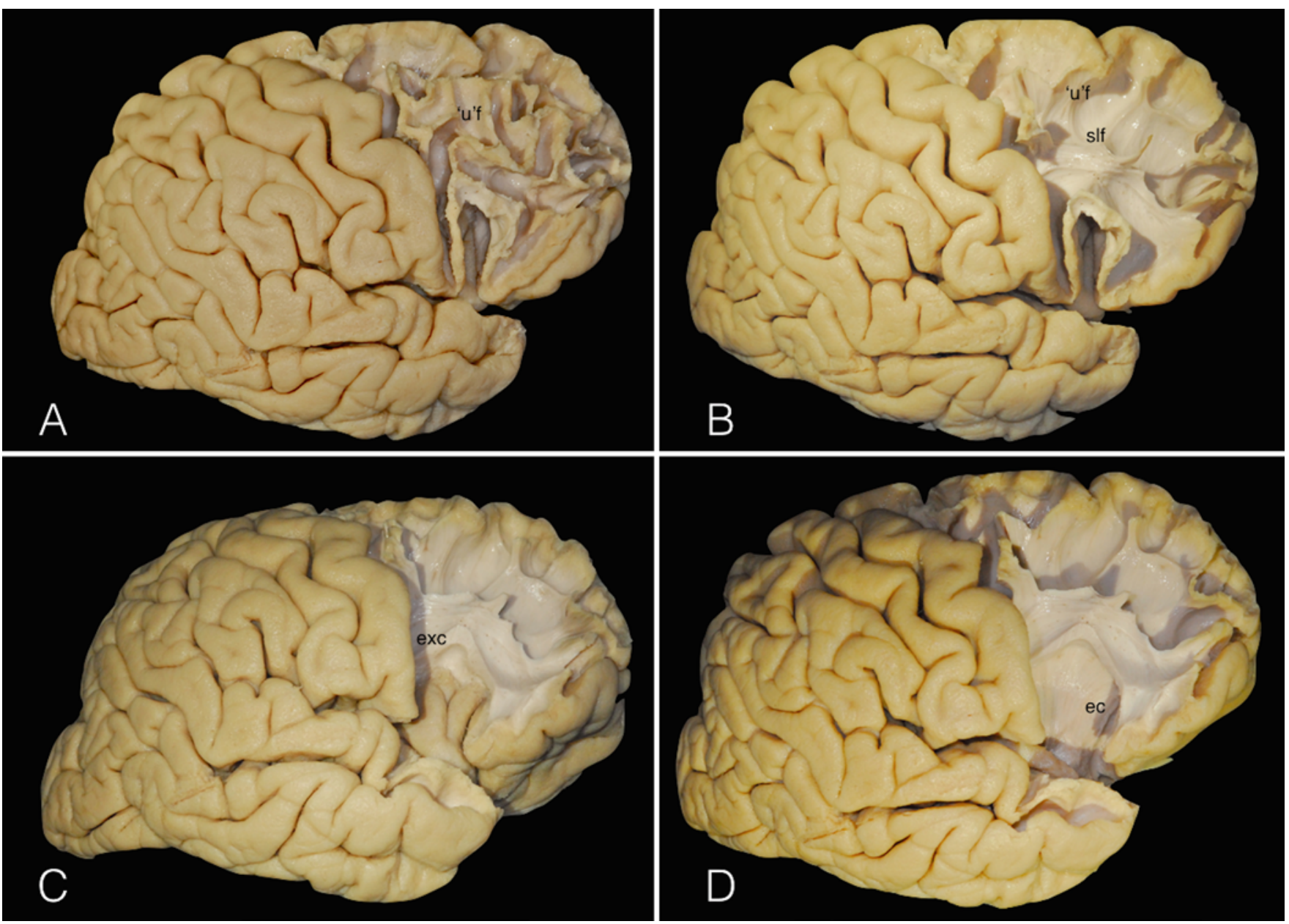

FIG. 2. High-definition images of a right hemisphere in which the first steps of the surgical procedure have been carried out.

A: After removal of the cortical gray matter, the first fiber tracts exposed are a group of short association or intergyral fibers, also known as $U$ fibers due to their shape joining adjacent gyri. These tracts have been exposed in the whole right frontal lobe between the superior, middle, and inferior frontal gyri. B: After removal of the $U$ fibers, the dorsal long association fiber system is exposed. This complex is represented by the rostral aspect of the superior longitudinal fascicle, which connects the frontal with the central, parietal, occipital, and temporal lobes. This white matter fiber tract runs deep to the middle frontal gyrus. C: The next step of the dissection is cutting of the inferior frontal gyrus stem to expose the insular surface as well as the short association insulo-opercular fibers, also known as the extreme capsule. The extreme capsule represents the short association fiber system of the insular lobe, connecting the short and long gyri with each other, and also projecting to the adjacent frontoorbital, frontoparietal and temporal operculi. D: After careful removal of the insular cortex and the extreme capsule, the first projection fiber system is exposed. The external capsule appears just medial to the extreme capsule and lies lateral to the basal ganglia. This fiber system joins the internal capsule at the dorsal aspect of the putamen. $\mathrm{ec}=$ external capsule; exc = extreme capsule; slf = superior longitudinal fasciculus; ' $u$ 'f $=$ short association fibers. 

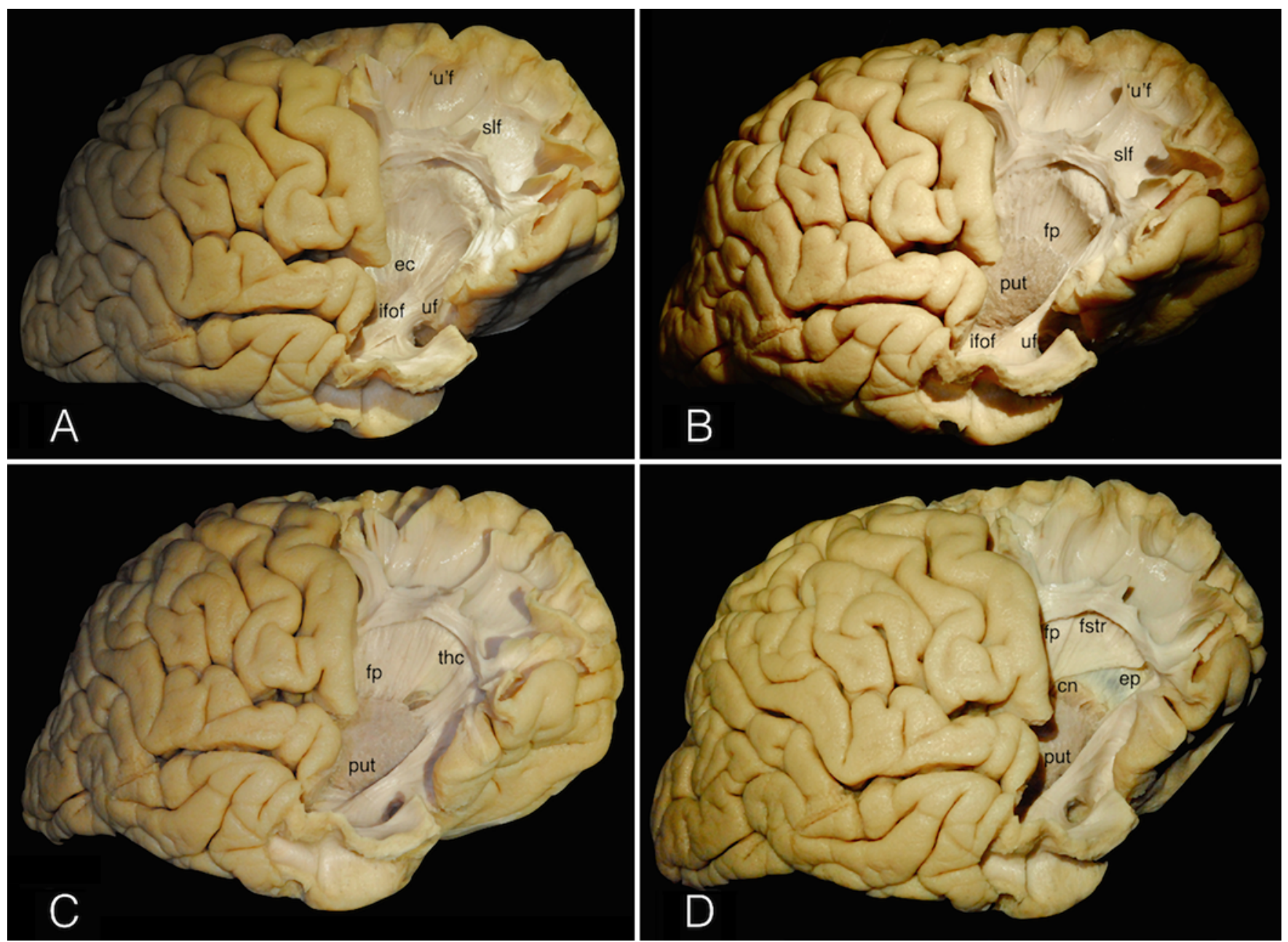

FIG. 3. High-definition images showing the next steps in the dissection of the right frontal and temporal lobes. A: The white matter dissection has now moved to the anterior aspect of the temporal lobe following 2 relevant association fiber systems. Thus, the uncinate and IFOF fibers represent the ventral long association fibers, which travel together at the level of the limen insula, just ventral to the claustrum and external capsule. The uncinate fascicle connects the fronto-orbital gyri together with the temporal pole and some temporomesial structures, such as some amygdalae nuclei. On the other hand, the IFOF connects the frontal pole and frontobasal structures with the occipital lobe running through the lateral aspect of the temporal lobe. B: The external capsule and the most dorsal aspect of the IFOF have been removed, uncovering the lateral surfaces of the putamen and the anterior limb of the internal capsule. The anterior limb of the internal capsule contains 3 types of projection groups of fibers. The most lateral ones are represented by the descending frontopontine fibers, which connect the frontal lobe with the pontine nuclei and middle cerebellar peduncle. These will be the first to be sectioned above the superior aspect of the putamen during the disconnection procedure. C: Removal of the lateral frontopontine fibers exposes the next group, which travels in the inner aspect of the internal capsule and is represented by the ascending thalamocortical fibers connecting the anterior thalamic nuclei and the prefrontal cortex (ascending fibers). D: Removal of the thalamocortical fibers uncovers the most medial aspect of the anterior limb of the internal capsule, which is mainly composed of the frontostriatal descending fibers. This bundle connects the frontal lobe with the striatum. These 3 bundles cross the striatum between the caudate nucleus and the putamen, creating a few gray matter bridges between them, which are called the striae. After removal of these fibers, the ependymal layer of the frontal horn of the lateral ventricle is exposed, giving access to the ventricular system. $\mathrm{cn}=$ head of the caudate nucleus; $\mathrm{ec}=$ external capsule; ep = ependymal layer of the lateral wall of the frontal horn; $f p=$ frontopontine fibers; $f s t r=$ frontostriate fibers; ifof = inferior fronto-occipital fascicle; put = putamen; slf = superior longitudinal fasciculus; thc = thalamocortical fibers; uf $=$ uncinate fascicle; ' $u$ ' $f=$ short association fibers

illustrate the white matter fascicles exposed and cut during the creation of the anterior suprainsular window and the intrafrontal disconnection (Fig. 2). Once the cortical gray matter is removed, the first fibers encountered are the short association fibers or $U$ fibers, which connect in this case the superior, middle, and inferior frontal gyri and the various portions of the inferior frontal gyrus (Fig. 2A).
After the removal of the U fibers, the dorsal long association fiber system is exposed. It consists of the superior longitudinal fasciculus (SLF), which runs deeply into the middle frontal gyrus and connects the frontal lobe with the precentral and postcentral sulcus and the parietal, occipital, and temporal lobes (Fig. 2B).

During the creation of the anterior suprainsular win- 
dow, a portion of the inferior frontal gyrus is removed in its opercular and triangular part to expose the anterosuperior part of the circular sulcus. The dissection is then continued in the depth of the sulcus, as it divides the anterior surface of the insular cortex from the posterior orbital gyrus on the surface. The first white matter tract encountered is the extreme capsule. This is composed of the short association insulo-opercular fibers, connecting the short with the long gyri of the insula itself and connecting the insula with the fronto-orbital, frontoparietal, and temporal opercula (Fig. $2 \mathrm{C}$ ). Continuing with the dissection, the external capsule is found medial to the extreme capsule (separated by the claustrum) and fuses with the internal capsule at the dorsal face of the putamen (Fig. 2D). Ventral to the claustrum and external capsule course the uncinate and the inferior fronto-occipital fasciculus (IFOF) (Fig. 3A and B). The uncinate fasciculus has a hook-like form and connects the fronto-orbital gyri with the temporal pole and the septal area (amygdaloid nuclei). The uncinate fasciculus courses inferior to the IFOF and deep to the limen insula. The IFOF connects the frontal pole and frontobasal gyri with the occipital lobe coursing along the temporal lobe (Fig. $3 \mathrm{~A}$ and $\mathrm{B}$ ) and may be divided into an anterior and posterior part according to the relationship with the circular sulcus. The anterior IFOF runs laterally to the inferior part of the frontal horn. The uncinate fasciculus and the IFOF are disconnected at their frontal projections as part of the suprainsular window step.

When the anterior suprainsular window is deepened to reach the frontal horn of the ventricle, the anterior limb of the internal capsule is disconnected. The anterior limb of the internal capsule contains 3 types of projection fibers. From lateral to medial these are the following: 1) the descending frontopontine fibers, connecting the frontal lobe with the pontine nuclei and middle cerebellar peduncle (Figs. 3C and 4); 2) the ascending thalamocortical fibers, connecting the anterior thalamic nuclei and the prefrontal cortex (Figs. 3C and 4); and 3) the frontostriatal descending fibers, connecting the frontal lobe with the striatum (Figs. 3D and 4). Once all these fibers are cut, the ependyma is visible and the frontal horn is entered into.

During the intrafrontal disconnection (Fig. 5), after cutting of the $\mathrm{U}$ fibers connecting the prefrontal cortex and the supplementary motor area with the precentral area, the horizontal portion of the SLF is sectioned (Fig. 3A). Then the corona radiata is sectioned until the interhemispheric fissure is reached (Fig. 5). During this stage, the main medial long association fibers are disconnected, represented by the cingulum. The short association fibers of the medial frontal lobe are also cut (Fig. 6).

\section{Medial Dissection}

Medial to lateral dissection was used to illustrate the white matter tracts sectioned during the anterior callosotomy (Fig. 6). The roof of the frontal horn of the lateral ventricle is formed by the anterior third of the body of the corpus callosum. The anterior wall is represented by the genu, while the floor is mainly formed by the callosal rostrum (Fig. 6B). During the anterior callosotomy, the cut is performed along the anterior third of the body of the callosum, the genu, and the rostrum to disconnect the

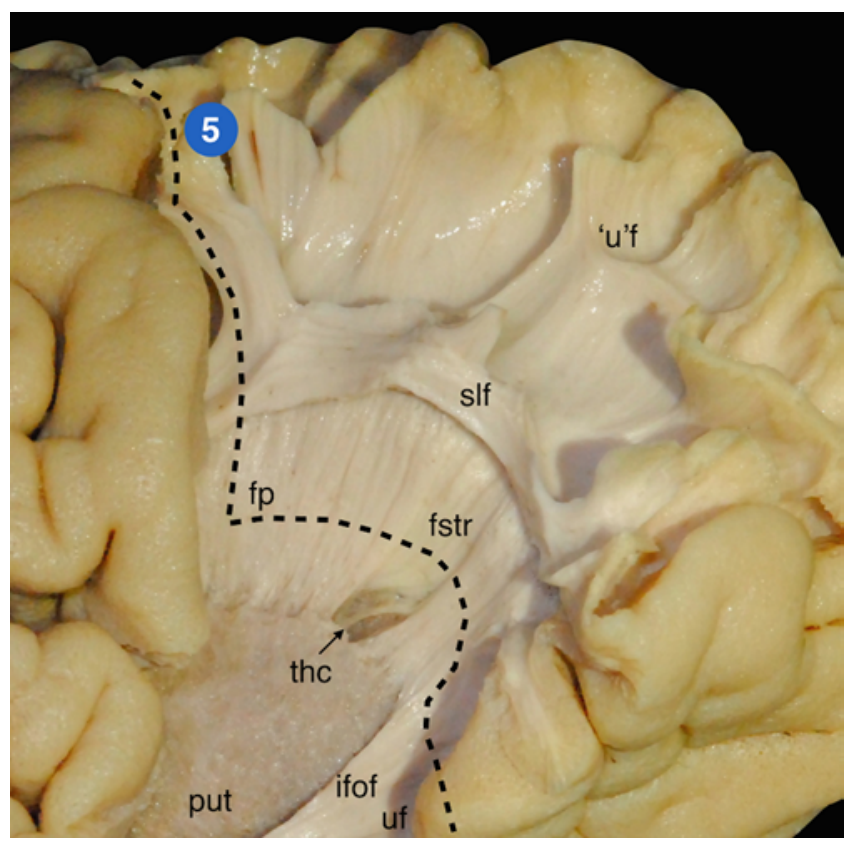

FIG. 4. In this high-definition dissection image, the 3 components of the anterior limb of the internal capsule are magnified, so its relative location is clearly exposed. From lateral to medial, the frontopontine, thalamocortical, and frontostriate bundles are sequentially dissected. The dashed line shows the section trajectory followed during surgery when approaching these deep regions. This fifth incision (5) will give us access to the medial aspect of the hemisphere superiorly and to the frontal horn of the lateral ventricle inferiorly after disconnection of the long association fiber systems (slf, uf, and ifof). Moreover, the internal capsule will be incised. Carrying out the inferior curve is important to avoid damaging the basal ganglia, represented here by the head of the caudate nucleus and the putamen. Sectioning inferiorly to the frontal horn gives access to the frontobasal regions, laterally disconnecting the frontal projections of the uncinate and inferior frontooccipital bundles. $\mathrm{fp}=$ frontopontine fibers; fstr = frontostriate fibers; ifof = inferior fronto-occipital fascicle; put = putamen; slf = superior longitudinal fasciculus; thc = thalamocortical fibers; $u f=$ uncinate fascicle; ' $u$ ' $f$ = short association fibers.

prefrontal area from the contralateral hemisphere (forceps minor) (Fig. 6). The fibers crossing in the ventral part of the callosum and connecting the caudate nuclei are also cut.

\section{Basal Dissection}

At the completion of the anterior suprainsular window, intrafrontal disconnection, and anterior callosotomy, the only remaining connections of the frontal lobe reside in the anterior commissure and short association fibers of the frontobasal area. These fibers are disconnected through the frontobasal disconnection, which is illustrated through medial and basal dissections (Figs. 7 and 8). The posterior portion of the frontobasal lobe is cut across the posterior part of the orbital and rectus gyri to reach the cut of the rostrum and thus the floor of the frontal horn. During this last step, the anterior limb of the anterior commissure is sectioned. This allows the disconnection between the fronto-orbital region and the subcallosal and septal areas and amygdala. ${ }^{7,9}$ 

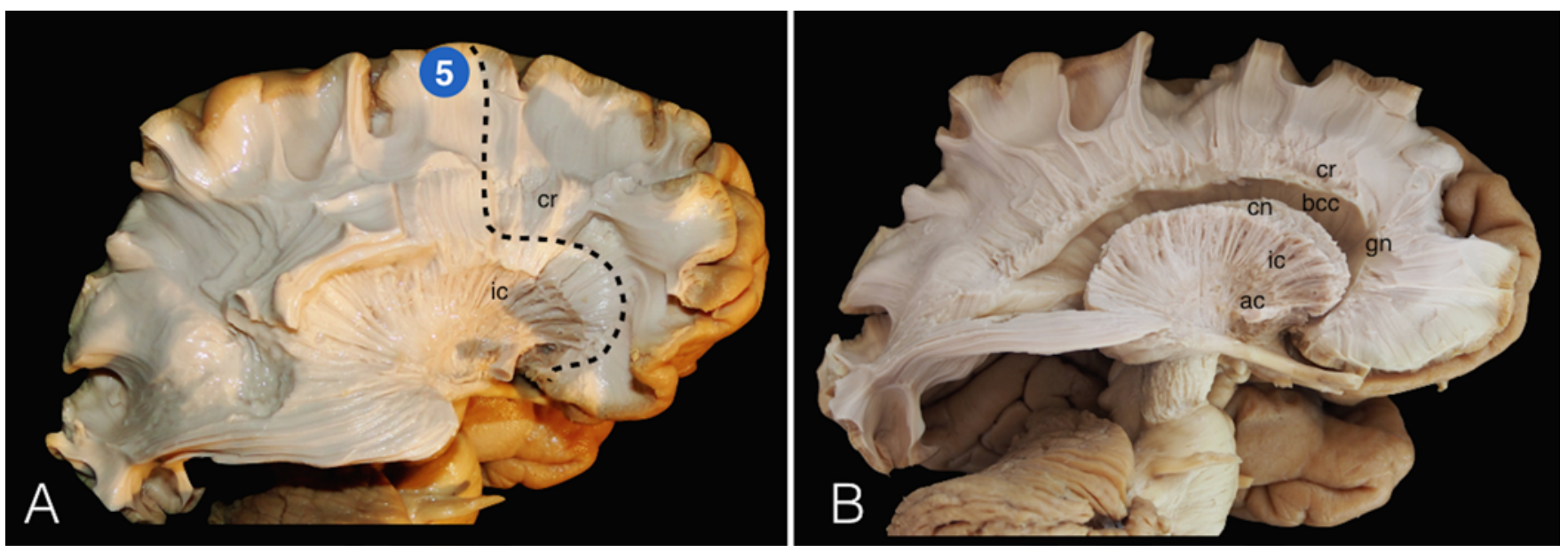

FIG. 5. A: The frontal projections of the superior longitudinal fascicle have been removed, showing the corona radiata and its continuity to the internal capsule after complete removal of the putamen and globus pallidus. The fifth section trajectory (5) is clearly exposed, showing how the internal capsule is fully disconnected at this step of the procedure. This maneuver will disconnect all the frontal lobe projection fibers. B: In this dissection, the internal capsule transition to the corona radiata has been intentionally opened, giving access to the lateral ventricle. Thus, the relationship between the caudate nucleus, lateral ventricle, projection fibers, and corpus callous can be clearly understood. The roof of the frontal horn of the lateral ventricle is composed of the anterior third of the body of the corpus callosum. The anterior wall is represented by the genu, while the floor is mainly formed by the callosal rostrum. The anterior commissure is another important commissural fiber system and is just anterior and ventral to the anterior limb of the internal capsule in this picture. The anterior commissure bilaterally connects some fronto-orbital regions, subcallosal and septal areas, and amygdala nuclei, as well as temporo- and occipitobasal regions. ac = anterior commissure; bcc = body of the corpus callosum; $\mathrm{cn}$ = caudate nucleus; $\mathrm{cr}=$ corona radiata; $\mathrm{gn}$ = genu of the corpus callosum; ic = internal capsule.

\section{Discussion}

Seizure freedom (Engel class I outcome) following frontal lobe epilepsy surgery is reported to occur in $45 \%$ of patients, according to a meta-analysis by Englot et al. ${ }^{7}$ This outcome is worse than the seizure outcome reported after temporal lobe epilepsy surgery. ${ }^{6-8}$ The seizure outcomes in patients undergoing frontal lobe epilepsy surgery are also quite variable between the reported studies, and good outcome rates vary between $20 \%$ and $78 \%{ }^{7}$ However, patients with small lesions seem to obtain better outcomes (52\%-54\% seizure free) ${ }^{7}$

The anterior quadrant disconnection represents an elegant technique for well-selected patients who present with large epileptogenic lesions well localized to the frontal lobe and situated anterior to the perirolandic cortex, and this procedure is therefore a valid alternate option to the classic frontal lobectomy. ${ }^{1,2}$ The principal objective of the surgery is the complete disconnection of the frontal lobe situated anterior to the primary motor cortex (anterior quadrant). To avoid motor deficits, preservation of the precentral gyrus and its projection, association, and commissural fibers is of primary importance during this surgery. Disconnective surgeries can be technically demanding due to the difficulty in perceiving the relevant anatomy, especially in cases where the normal anatomy is distorted. Sequential cadaveric dissections simulating the different steps of the procedure allow a thorough understanding of the 3D anatomy of this region, as shown in this article through high-definition white matter dissection images. Anatomical landmarks as described in this study are invaluable to guide the surgery. The use of these surgical landmarks should obviously be combined with intraoperative electrophysiological studies. Somatosensory evoked potentials are used to localize the central sulcus and guide the cortical incision anterior to the motor strip during the intrafrontal step. Motor stimulation is used over the cortex to confirm the localization of the central sulcus and can also be used to stimulate the corona radiata, to prevent damage to projection fibers from the motor cortex. ${ }^{1}$ The callosotomy should include only the anterior third of the callosum (the anterior part of the body, genu, and rostrum) and should spare the callosal fibers of the parietal and occipital lobes. Whether the disconnection is complete can be determined on continuous EEG monitoring, where it is evidenced by the elimination of epileptiform discharges that propagate from the epileptogenic frontal cortex to the ipsilateral posterior quadrant and to the contralateral hemisphere.

Compared with resective procedures, disconnective procedures in general have some advantages for large hemispheric or subhemispheric epilepsy surgery. ${ }^{3-5}$ Removal of large amounts of brain tissue during resection, such as in hemispherectomy or quadrantectomy, has been known to produce "cavity complications" like aseptic meningitis, hydrocephalus, and superficial hemosiderosis. ${ }^{4}$ These complications have markedly decreased in incidence following the use of disconnective procedures for these epileptic syndromes. ${ }^{9}$ In addition, the craniotomy for a disconnective procedure is smaller than that for a resective procedure, as disconnective procedures do not require exposure of the skull base and major venous sinuses. Thereby the craniotomy-related morbidity is lessened and the postoperative management becomes easier. However, 

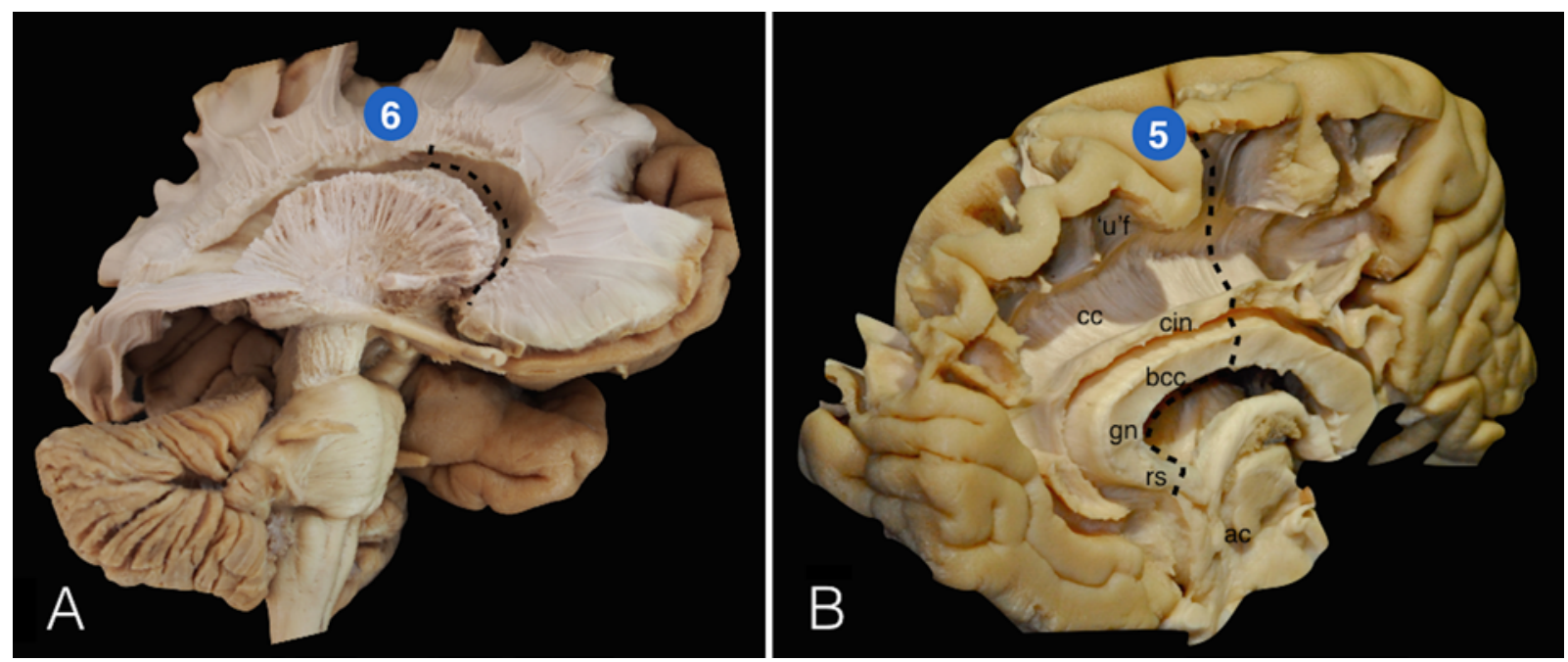

FIG. 6. A: The sixth incision (6) is represented by a dashed line. At this stage the intraventricular work is carried out, disconnecting the main commissural fibers, which are represented by the anterior third of the body of the corpus callosum, the genu, and the rostrum. This maneuver will disconnect the lateral and medial aspects of the frontal lobe bilaterally. However, the frontobasal, septal, and subcallosal areas of the frontal lobe still remain connected bilaterally by the anterior limb of the anterior commissure. B: The fifth incision (5), performed in previous stages, not only accesses the frontal horn, but also should move us to medial aspect of the hemisphere. Thus the disconnection at this stage will start sectioning the main medial long association fiber system, which is represented by the cingulum, and later on will disconnect the short association fibers of the medial frontal lobe. Once the fifth and sixth maneuvers are completed, the only remaining connections of the frontal lobe are intact through the anterior commissure and short association fibers of the frontobasal area. $\mathrm{ac}=$ anterior commissure; bcc = body of the corpus callosum; $c c=$ corpus callosum; $\mathrm{cin}$ = cingulum fibers; gn = genu of the corpus callosum; rs = rostrum of the corpus callosum; 'u' $f$ = short association fibers of the medial frontal lobe.

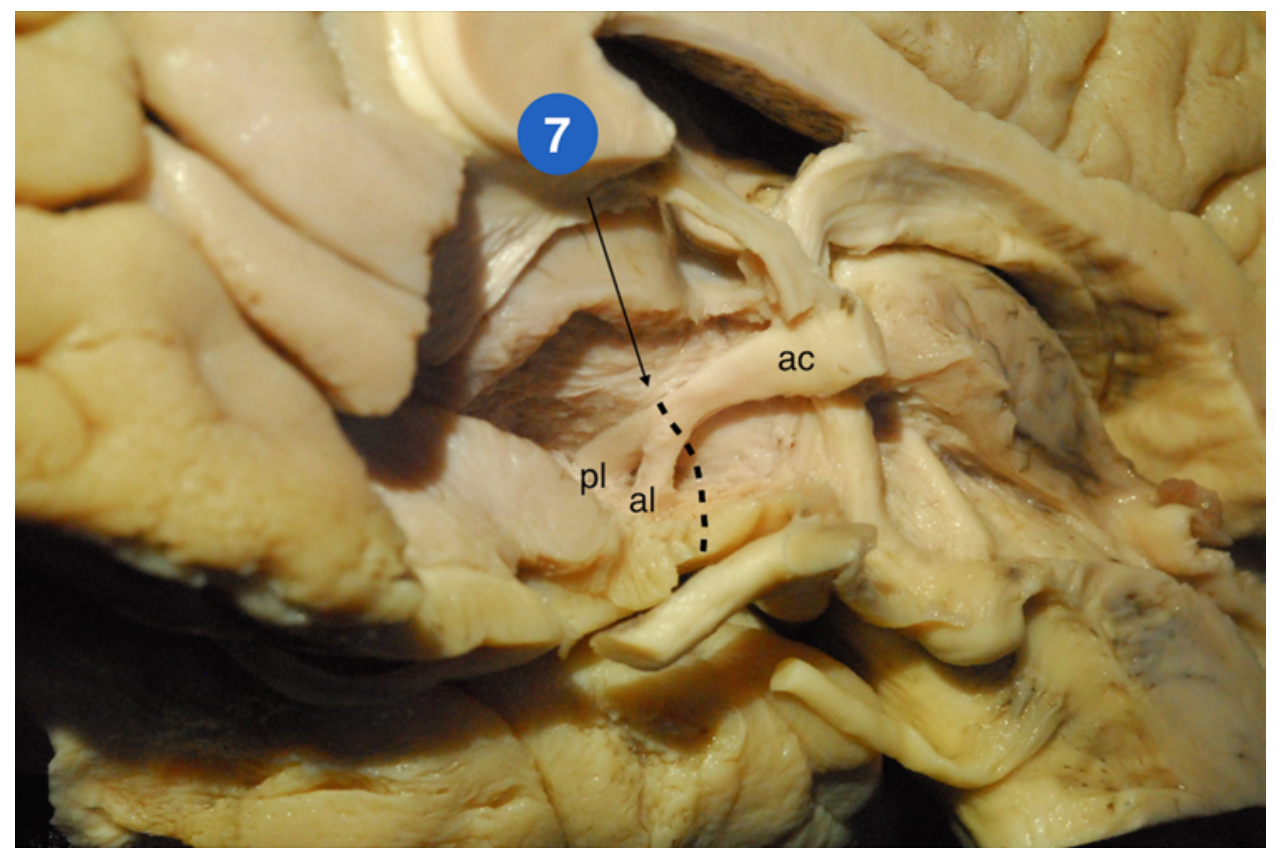

FIG. 7. High-definition dissection image of a right hemisphere from a laterobasal view, where the subcallosal, septal, posterior part of the gyrus rectus and posteromedial frontoorbital areas have been dissected to show the anterior and posterior limbs of the anterior commissure. The anterior limb of the anterior commissure bilaterally connects some fronto-orbital regions, the subcallosal and septal areas, and some amygdala nuclei, while the posterior limb does so with the temporo- and occipitobasal regions of both hemispheres. The main goal of a full anterior disconnection at this stage is focused on the seventh section trajectory (7), which is directed posterobasally from the floor of the frontal horn to the posterior aspect of the gyrus rectus. This trajectory will fully disconnect this anterior limb of the anterior commissure. $a c=$ anterior commissure; $a l=$ anterior limb of the anterior commissure; $\mathrm{pl}=$ posterior limb of the anterior commissure. 


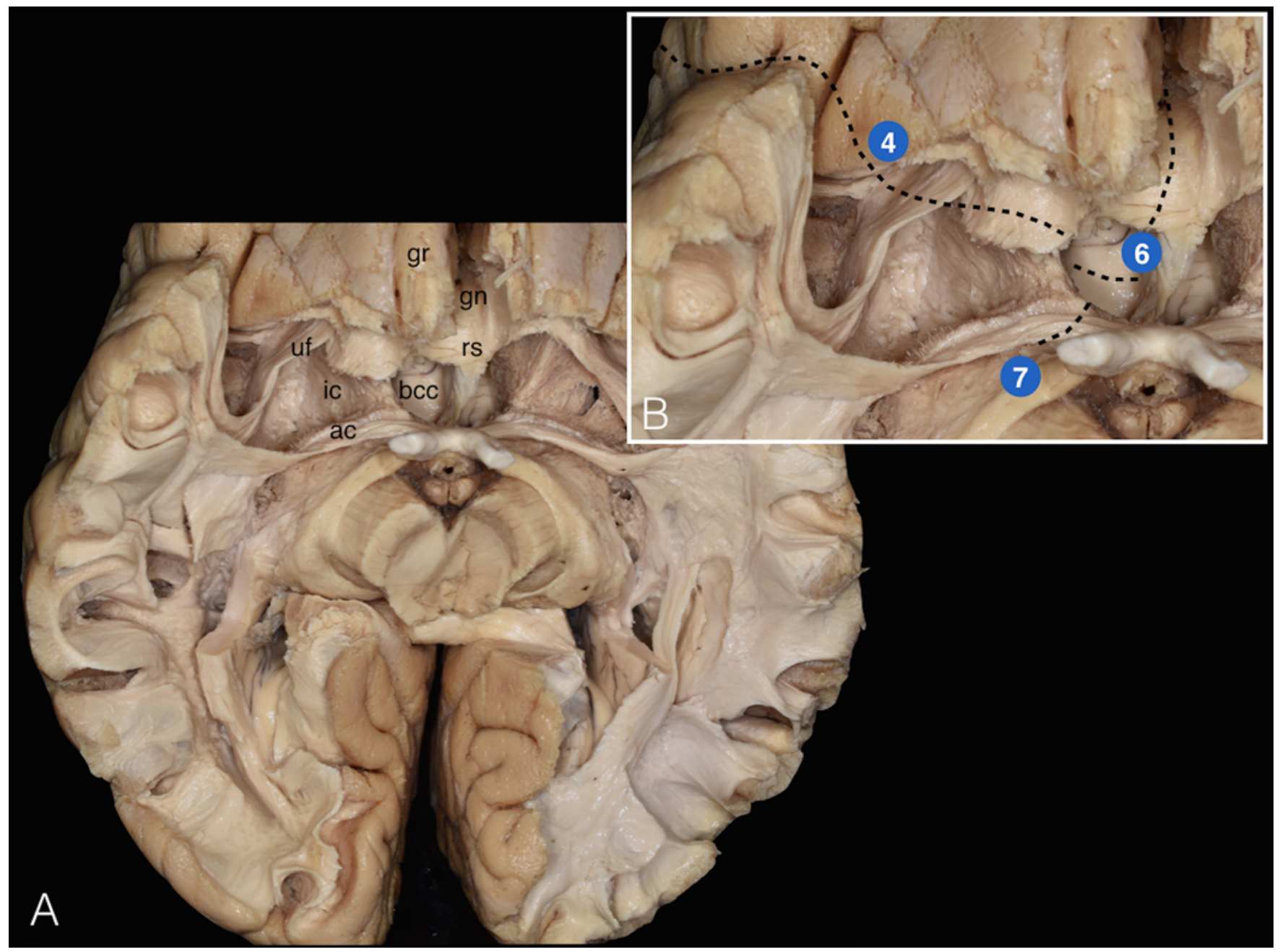

FIG. 8. A: Basal view of both cerebral hemispheres. The short association fibers as well as the posterior third of the rectus gyri, basal ganglia, and septal and subcallosal areas have been resected. The basal aspect of the frontal horns of the lateral ventricles are shown after removal of the rostrum of the corpus callosum. The anterior commissure and its relationship to the uncinate fascicle and the basal aspect of the frontal lobes are exposed. B: The fourth corticotomy (4) is represented crossing the posterior aspect of the frontobasal lobe connecting with the floor of the frontal horn. The sixth white matter transection (6) can be understood when seen from below, disconnecting the anterior third of the body and genu of the corpus callosum. The seventh and last cut (7) is seen from below, disconnecting the mesial aspect of the anterior commissure, mainly its anterior limb. For the surgical procedure in patients, the frontobasal disconnection, which represents the final part of the surgical disconnection, begins with the incision of the frontal pial convexity at the level of the lateral aspect of the pars orbitalis. This incision is deepened in the white matter in a direction parallel to the sphenoid ridge. The posterior fronto-orbital region and gyrus rectus are cut in a subpial fashion by continuing from the earlier pial convexity incision. The basal pia is left uncut and the olfactory tract visualization is a good landmark for orientation. This subpial disconnection reaches the interhemispheric fissure, where the mesial aspects of the gyrus rectus, anterior cingulate, and subcallosal areas are interrupted in a subpial fashion. During this stage the A2 arteries (one or both) can be seen through the pia and offer additional anatomical confirmation. This gyral section, when deepened, accesses the floor of the frontal horn where it meets the most proximal end of the callosal disconnection (rostrum). ac = anterior commissure; bcc = body of the corpus callosum; gn = genu of the corpus callosum; gr = gyrus rectus; ic = internal capsule; $r s=$ rostrum of the corpus callosum; uf = uncinate fasciculus.

the disconnective procedure for frontal lobe epilepsy is a new procedure for a rare disease and to our knowledge, apart from our earlier description, ${ }^{3}$ outcome data on this procedure are unavailable in literature.

\section{Conclusions}

The anterior peri-insular quadrantotomy is a surgical technique that allows effective treatment of patients with a large refractory epileptogenic area localized to the frontal lobe anterior to the motor cortex. Precise understanding of the gyral anatomy of this lobe and of the main projection and associative fibers, along with knowledge of the commissural white matter tracts, are crucial to achieve total isolation of this lobe and at the same time to avoid postoperative motor deficits. A precise knowledge of the mi- 
crosurgical and white matter anatomy is essential to safely perform the surgery with the aim of a complete disconnection and to ensure the highest chance of seizure freedom in this population of pediatric patients with intractable anterior quadrant epilepsy.

\section{References}

1. Asa SL, Casar-Borota O, Chanson P, Delgrange E, Earls P, Ezzat S, et al: From pituitary adenoma to pituitary neuroendocrine tumor (PitNET): an International Pituitary Pathology Club proposal. Endocr Relat Cancer 24:C5-C8, 2017

2. Cossu G, Lebon S, Seeck M, Pralong E, Messerer M, RouletPerez E, et al: Periinsular anterior quadrantotomy: technical note. J Neurosurg Pediatr 21:124-132, 2018

3. Daniel RT, Babu KS, Jacob R, Villemure J: Posterior quadrantic resection and disconnection, in Cataltepe O, Jallo GI (eds): Pediatric Epilepsy Surgery: Preoperative Assessment and Surgical Treatment. New York: Thieme Medical Publishers, 2010, pp 196-204

4. Daniel RT, Joseph TP, Gnanamuthu C, Chandy MJ: Hemispherotomy for paediatric hemispheric epilepsy. Stereotact Funct Neurosurg 77:219-222, 2001

5. Daniel RT, Meagher-Villemure K, Farmer JP, Andermann F, Villemure JG: Posterior quadrantic epilepsy surgery: technical variants, surgical anatomy, and case series. Epilepsia 48:1429-1437, 2007

6. Elsharkawy AE, Alabbasi AH, Pannek H, Schulz R, Hoppe M, Pahs G, et al: Outcome of frontal lobe epilepsy surgery in adults. Epilepsy Res 81:97-106, 2008

7. Englot DJ, Wang DD, Rolston JD, Shih TT, Chang EF: Rates and predictors of long-term seizure freedom after frontal lobe epilepsy surgery: a systematic review and meta-analysis. J Neurosurg 116:1042-1048, 2012

8. Jeha LE, Najm I, Bingaman W, Dinner D, Widdess-Walsh P, Lüders H: Surgical outcome and prognostic factors of frontal lobe epilepsy surgery. Brain 130:574-584, 2007
9. Rumalla K, Smith KA, Follett KA, Nazzaro JM, Arnold PM: Rates, causes, risk factors, and outcomes of readmission following deep brain stimulation for movement disorders: analysis of the U.S. Nationwide Readmissions Database. Clin Neurol Neurosurg 171:129-134, 2018

10. Salanova V, Quesney LF, Rasmussen T, Andermann F, Olivier A: Reevaluation of surgical failures and the role of reoperation in 39 patients with frontal lobe epilepsy. Epilepsia 35:70-80, 1994

11. Téllez-Zenteno JF, Dhar R, Wiebe S: Long-term seizure outcomes following epilepsy surgery: a systematic review and meta-analysis. Brain 128:1188-1198, 2005

\section{Disclosures}

The authors report no conflict of interest concerning the materials or methods used in this study or the findings specified in this paper.

\section{Author Contributions}

Conception and design: Daniel, Cossu. Acquisition of data: Daniel, Gonzalez-Lopez, Cossu. Analysis and interpretation of data: Daniel, Gonzalez-Lopez, Cossu, Baldoncini, Messerer. Drafting the article: Gonzalez-Lopez, Cossu, Pralong, Messerer. Critically revising the article: Daniel, Gonzalez-Lopez, Cossu, Pralong, Messerer. Reviewed submitted version of manuscript: Daniel, Gonzalez-Lopez, Cossu, Messerer. Approved the final version of the manuscript on behalf of all authors: Daniel. Administrative/ technical/material support: Gonzalez-Lopez, Baldoncini. Study supervision: Daniel.

\section{Correspondence}

Roy Thomas Daniel: University Hospital of Lausanne, Switzerland.roy.daniel@chuv.ch. 